\title{
Eliminating the attentional blink through binaural beats: a case for tailored cognitive enhancement
}

\author{
Susan A. Reedijk, Anne Bolders, Lorenza S. Colzato and Bernhard Hommel* \\ Institute for Psychological Research, Leiden Institute for Brain and Cognition, Leiden University, Leiden, Netherlands
}

Enhancing human cognitive performance is a topic that continues to spark scientific interest. Studies into cognitive-enhancement techniques often fail to take inter-individual differences into account, however, which leads to underestimation of the effectiveness of these techniques. The current study investigated the effect of binaural beats, a cognitiveenhancement technique, on attentional control in an attentional blink (AB) task. As predicted from a neurocognitive approach to cognitive control, high-frequency binaural

OPEN ACCESS

Edited by:

Leila Chaieb,

University of Bonn, Germany

Reviewed by:

Philip R. Corlett,

Yale School of Medicine, USA

Wi Hoon Jung,

University of Pennsylvania, USA Andrew Watrous,

University of California Davis, USA

*Correspondence:

Bernhard Hommel,

Cognitive Psychology Unit, Leiden

University, Wassenaarseweg 52,

Leiden 2333 AK, Netherlands

hommel@fsw.leidenuniv.nI

Specialty section:

This article was submitted to

Neuropsychiatric Imaging and

Stimulation, a section of the journal

Frontiers in Psychiatry

Received: 22 December 2014

Accepted: 18 May 2015

Published: 04 June 2015

Citation:

Reedijk SA, Bolders A, Colzato LS and Hommel B (2015) Eliminating

the attentional blink through

binaural beats: a case for tailored

cognitive enhancement.

Front. Psychiatry 6:82.

doi: 10.3389/fpsyt.2015.00082 beats eliminated the $A B$, but only in individuals with low spontaneous eye-blink rates (indicating low striatal dopamine levels). This suggests that the way in which cognitiveenhancement techniques, such as binaural beats, affect cognitive performance depends on inter-individual differences.

Keywords: attentional blink, dopamine, attention, binaural beats, neurotransmitters, cognitive enhancement

\section{Introduction}

For ages, humans have used cognitive and physical interventions to improve their performance, and new cognitive-enhancement techniques are announced daily. Unfortunately, most of them do not stand scientific evaluation (1), with the recent failure to find an effect of brain training in more than 11,000 participants (2) being a particularly attention-grabbing example. And yet, many tests are likely to systematically underestimate the potential of enhancement techniques by ignoring inter-individual differences in cognitive or neural parameters that determine or reflect individual sensitivity to interventions.

One indication that individual differences play an important role in the degree to which enhancement techniques affect cognition is the observation that the widely assumed creativityenhancing effect of positive mood (3) is mediated by individual differences related to dopamine levels. As demonstrated by Akbari Chermahini and Hommel (4), individuals with low dopamine levels (as assessed by spontaneous eye-blink rates; EBRs) show better performance in a divergentthinking task after the induction of positive mood, while individuals with medium or high dopamine levels show no effect. Accordingly, studies that neglect functionally relevant individual differences can be expected to replicate or fail to replicate the assumed connection between creativity and mood, depending on the specific characteristics of the given sample - which explains the great inconsistency between the available studies [see Ref. (3)].

The present study assessed an enhancement technique that has been claimed to target cognitivecontrol functions: binaural beats - the subjective experience of a beating tone with a frequency that corresponds to the frequency difference between two binaurally presented tones (5). As first reported by Heinrich Wilhelm Dove in 1839, presenting two sounds of slightly different frequencies (330 and $350 \mathrm{~Hz}$, say) to the left and right ear results in the integrated perception of one sound that fluctuates in 
a frequency that corresponds to the difference $(20 \mathrm{~Hz})$. While the neural mechanisms underlying this illusion remain unknown, a role of the reticular activation system has been proposed (6). In animals, binaural beat-inducing conditions are associated with spreading neural patterns of phase locking in the auditory cortex, which ultimately result in specific firing patterns in the inferior colliculus that could reflect a binaural beat $(7,8)$.

Even though weaker than the neural response to beats physically present in the signal (9), binaural beats elicit a similar neural response as real acoustic beats $(7,8,10,11)$. This suggests that the illusion of a beating tone arises through neural pathways normally associated with binaural sound detection in the environment $(7,9)$. In humans, the neural phase locking that binaural beats elicit can influence ongoing cognitive processing. Lowfrequency binaural beats are associated with mental relaxation and high-frequency beats with alertness and attentional concentration $(1,6)$. This suggests that high-frequency beats might facilitate attentional control, which would fit with the observation that high-frequency neurofeedback training over the frontal cortex improves attentional efficiency (12).

We tested this hypothesis by using the attentional blink (AB) task, in which two targets (T1 and T2) are presented in a rapid stimulus stream. While participants are commonly able to report T1, they often miss T2 if it appears soon after T1 [(13); for a review, see Ref. (14)]. While factors such as T1 target identification, consolidation, and response selection also play a role (15), the $\mathrm{AB}$ mainly has been attributed to the suboptimal allocation of attentional resources to the two targets: too much emphasis on T1 leaves too few resources for T2 (16-19). Indeed, interventions that supposedly improve attentional control, like meditational practice, have been shown to reduce the $\mathrm{AB}(20)$.

Cognitive-control functions, such as those needed in the $A B$ task, rely on the interplay between two dopaminergic pathways: a frontal pathway driving working memory and top-down control functions, and a striatal pathway supporting mental flexibility and bottom-up interruptions (21). Interestingly for our purposes, people can be biased toward one or the other pathway. Those who are biased toward the frontal dopamine pathway show high prefrontal but low striatal dopamine levels, while those biased toward the striatal pathway show evidence of low prefrontal but high striatal dopamine levels [for reviews, see Ref. $(22,23)]$ - with the latter being associated with higher spontaneous eye-blink rates [EBRs; see Ref. $(24,25)]$. Also of interest, individual differences in the balance between these two pathways in the corresponding functions have been demonstrated to modulate the size of the $A B$ : it is smaller in individuals with high working-memory span (26) a frontal function (21) - and with a genetic setup that is associated with high frontal and low striatal dopamine levels (27).

In the present study, we used EBR, a well-established clinical indicator (28) thought to index dopamine (DA) production in the striatum (29-31), to assess individual (striatal) dopaminergic functioning. The idea that EBR mirrors dopaminergic functioning is first of all supported by clinical observations in patients with DA-related dysfunctions. For example, EBRs are elevated in schizophrenia patients (32), who in PET studies showed elevated striatal dopamine uptake, both on and off medication $(33,34)$. By contrast, EBRs are reduced in recreational cocaine users (35), and in Parkinson's patients (36) - two populations suffering from reduced functioning of D2 receptors and severe losses of nigrostratial dopaminergic cells, respectively $(37,38)$. Further, Colzato et al. (39) showed that the level of Psychoticism, which has been associated with dopaminergic activity (40), was predicted by EBR: people with higher scores on the Psychoticism scale showed higher EBRs. Second, pharmacological studies in non-human primates and humans have shown that DA agonists, as apomorphine, and antagonists increase and decrease EBRs, respectively $(29,41)$. Third, a genetic study in humans demonstrated a strong association between EBR and the DRD4/7 genotype, which is related to the control of striatal DA release (24). Above all, a recent study employing PET (42) has shown that baseline EBR was positively related to striatal D2-like receptor availability throughout the striatum. Accordingly, we assumed EBR to indicate an individual bias toward the frontal (low EBR) or the striatal pathway (high EBR).

How might individual differences related to the striatal dopamine level modulate enhancement effects? Given the evidence that high frontal and low striatal dopamine levels are associated with better performance the $\mathrm{AB}$ task (27), enhancing cognitive control would be expected to induce or increase the bias toward the frontal pathway. It might seem obvious to assume that the benefits are strongest for those who need them most, that is, for individuals with a bias toward the striatal pathway (i.e., with higher EBRs). As these individuals are more drawn to cognitive flexibility than to cognitive control, a stimulus that enhances cognitive control might affect them more than individuals who already favor control over flexibility. However, recent enhancement studies with genetic predictors suggest that more reliable enhancement can be found in individuals with a more suitable predisposition [i.e., are, genetically or otherwise, predisposed toward the cognitive process(es) the present task calls for; (43)], suggesting that more enhancement might be found in individuals with a stronger bias toward the frontal cognitivecontrol pathway (i.e., individuals with lower EBRs). We tested these possibilities by comparing $\mathrm{AB}$ performance in individuals with low and high EBRs, during exposure to binaural beats with low (alpha) or high (gamma) frequency, or a constant tone as control.

\section{Materials and Methods}

Twenty-four students (22 female, 2 male; aged 17-25 years old) of Leiden University participated in this study in exchange for course credit or pay. All had normal or corrected-to-normal sight and hearing. Participants were considered suitable to participate in this study if they fulfilled the following criteria: (i) age between 17 and 30 years; (ii) no history of neurological or psychiatric disorders; (iii) no history of substance abuse or dependence. After the study was explained to them by the experimenter, written informed consent was obtained from all participants. In the case of one underage participant, written informed consent was also obtained from their parents/caretakers. The study was approved by the Leiden University Ethics Committee of the Institute of Psychology. 
As mood correlates with dopamine levels (4), we controlled for mood by means of a $9 \times 9$ arousal/pleasure affect grid (44). Participants took part in three sessions in counterbalanced order, where they listened to alpha-frequency $(10 \mathrm{~Hz})$ or gamma-frequency $(40 \mathrm{~Hz})$ binaural beats, or a constant tone of $340 \mathrm{~Hz}$ (control condition), all embedded in white noise to enhance clarity of the beats (5), for 3 min before and during the AB task. Binaural beats were presented through in-ear headphones (Etymotic Research ER-4B microPro), which provide $35 \mathrm{~dB}$ noise attenuation. Both binaural beat conditions were based on a $340 \mathrm{~Hz}$ carrier tone, which was used as the constant tone in the control condition. EBRs were measured at the beginning of each session for $5 \mathrm{~min}$ before presentation of the binaural beats, using six $\mathrm{Ag} / \mathrm{AgCl}$ electrodes: two placed next to each eye (measuring saccades), and two placed above and below the right eye (measuring the blink). Two electrodes placed on the mastoids served as a reference. Participants were instructed to relax and look (but not stare!) straight ahead at a paper with a fixation cross that was taped to the computer monitor. This monitor was turned off during the EBR measurement. As EBR is stable during the day but goes up in the evening, participants were never tested after 7 p.m. (45). Before analysis, the three EBR measurements were averaged to create one stable EBR count for every participant.

In the $\mathrm{AB}$ task, two digit targets (drawn from 2 to 9) and 18 letter distracters (randomly drawn from A to Z) were presented for $70 \mathrm{~ms}$ each with an inter-stimulus interval of $30 \mathrm{~ms}$ at the center of a 19-inch CRT monitor $(1024 \times 768$ pixels, $100 \mathrm{~Hz}$ vertical refresh rate). To reduce predictability, the position of $\mathrm{T} 1$ in the rapid serial visual presentation (RSVP) stream varied randomly between positions 7, 8, and 9. Participants were to report T1 before $\mathrm{T} 2$ by pressing the corresponding number keys on a keyboard. T2 appeared for 100, 300, 500, and $800 \mathrm{~ms}$ after T1 (lags 1, 3, 5, and 8 , respectively).

\section{Results}

As a repeated measures analysis of variance (ANOVA) did neither find any differences in pre-experimental EBR between sessions, $F(2,46)=1.23, p=0.303, \eta_{\mathrm{p}}^{2}=0.05$, nor between binaural beat conditions, $F(2,46)=1.77, p=0.18, \eta_{\mathrm{p}}^{2}=0.07$, two groups of 12 participants each were created by median (17.4) split of the average EBR score over all three sessions. The groups' mood did not differ in both arousal or pleasure, $F s(2,22)<1$. A mixed ANOVA (Greenhouse-Geisser corrected where necessary) of conditional T2 performance (T2|T1) yielded a three-way interaction between EBR group, stimulation, and lag, $F(3.04,66.95)=3.38, p=0.023$, $\eta_{\mathrm{p}}^{2}=0.13$. Separate analyses revealed lag effects in the lowEBR group, $F(1.28,14.03)=9.35, p=0.006, \eta_{\mathrm{p}}^{2}=0.46$, and the high-EBR group, $F(1.35,14.85)=10.33, p=0.003, \eta_{\mathrm{p}}^{2}=$ 0.48 , reflecting the standard $\mathrm{AB}$ pattern. This effect interacted with stimulation in the low-EBR group, $F(2.52,27.66)=3.59$, $p=0.032, \eta_{\mathrm{p}}^{2}=0.25$, but not in the high-EBR group, $F(2.73$, 29.99) $<1$.

As Figure 1 suggests, the interaction in the low-EBR group was produced by the gamma condition (and disappeared in an ANOVA without that condition, $p>0.58$ ), where the $\mathrm{AB}$ was no longer reliable, $p>0.1$. To get a better view of the relationship between EBR and gamma-frequency stimulation, we calculated a gamma benefit variable by subtracting lag 3 accuracy in the control condition from lag 3 accuracy in the gamma condition, and entered these variables in a regression analysis. Given that cognitive performance has been shown to relate to EBR in nonlinear ways [leading to quadratic relationships: (46)], we computed both linear and quadratic fits. As shown in Figure 2, there exists a positive quadratic relationship between EBR and benefit from gamma stimulation on lag $3, F(2,21)=3.84, p=0.038$, while the linear relationship failed to reach significance, $F(1,22)=3.89$, $p=0.061$. The same analysis was done with an alpha benefit variable, but neither the linear, $F(1,22)=1.03, p=0.32$, nor the quadratic relationship, $F(2,21)<1$, reached significance ${ }^{1}$.

\section{Conclusion}

As expected, we found evidence for the enhancement of attentional control through high-frequency binaural beats. Given that the $A B$ is considered a very robust effect, it is remarkable that gamma beats eliminated this effect altogether. Importantly, the success of the enhancement intervention was predicted by EBR, our marker of the individual striatal dopamine level. While no enhancement was observed for people with high EBRs (which we take to indicate a bias toward the striatal pathway), reliable enhancement was obtained in individuals with low EBRs (presumably indicating a bias toward the frontal pathway). This suggests that enhancement techniques like binaural beats are unable to compensate for unfavorable control styles or dispositions but rather further improve individuals that have a suitable control style or disposition in place already - a conclusion that fits with the implications of a recent training study (43).

A possible explanation for why gamma-frequency entrainment eliminates the $\mathrm{AB}$ in individuals with a low EBR could be that presenting these individuals with gamma binaural beats leads to temporarily increased activity in the gamma band during presentation of $\mathrm{T} 1$ and $\mathrm{T} 2$, inside and outside of the AB period. Normally, increased gamma synchronization on the EEG only occurs after presentation of $\mathrm{T} 1$ and after presentation of $\mathrm{T} 2$ outside of the $\mathrm{AB}$ period, while this gamma peak does not occur after presentation of T2 inside the AB period [300 ms after presentation of T1; (47)]. This suggests that, at least in people with an already favorable cognitive control style, gamma-frequency binaural beats enhance gamma synchronization in neural firing, not only on non- $A B$ trials but also during the sensitive $\mathrm{AB}$ period.

Another possible explanation is that the gamma beat successfully distracts individuals who otherwise would allocate too many resources to T1 (16-19). If individuals with a low EBR (who most likely favor the frontal dopamine pathway) are more sensitive to the distracting gamma-frequency beat, they would indeed show a larger decrease in $\mathrm{AB}$ than individuals with high EBRs (who likely

\footnotetext{
${ }^{1}$ Given that Colzato et al. (52) reported higher ABs for low blinkers, we also tested for this effect by calculating the maximal $\mathrm{AB}$ in the Control condition for each participant by using Colzato et al.s formula (T2|T1 at Lag 8 minus the minimum of T2|T1 at Lag3 and at Lag 5) and compared it across the two EBR groups. Numerically, the AB was indeed larger for low blinkers (0.13) than for high blinkers (0.08), but this effect was far from significant, $t(22)<1$, suggesting that more power might be needed to obtain a stable effect of that sort.
} 


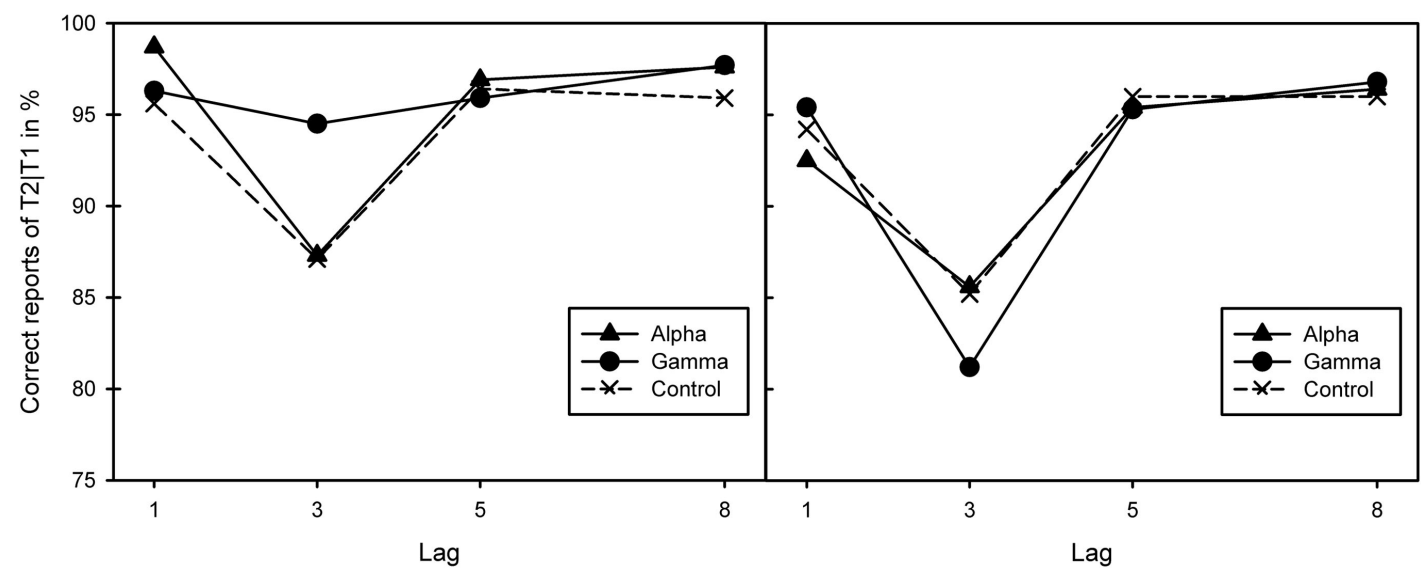

FIGURE 1 | Correct report of T2|T1 for the three binaural beat conditions (alpha, gamma, and control) in the two EBR groups. Lag effects in both the low [left-hand graph; $F(1.28,14.03)=9.35$, $\left.p=0.006, \eta_{p}^{2}=0.46\right]$ and high-EBR groups [right-hand graph; $F(1.35$,
$14.85)=10.33, p=0.003, \eta_{\mathrm{p}}^{2}=0.48$ reflective of the standard attentional blink pattern. This interacted with stimulation in the low-EBR group $[F(2.52$, $\left.27.66)=3.59, p=0.032, \eta_{p}^{2}=0.25\right]$ but not in the high-EBR group $[F(2.73,29.99)<1]$.

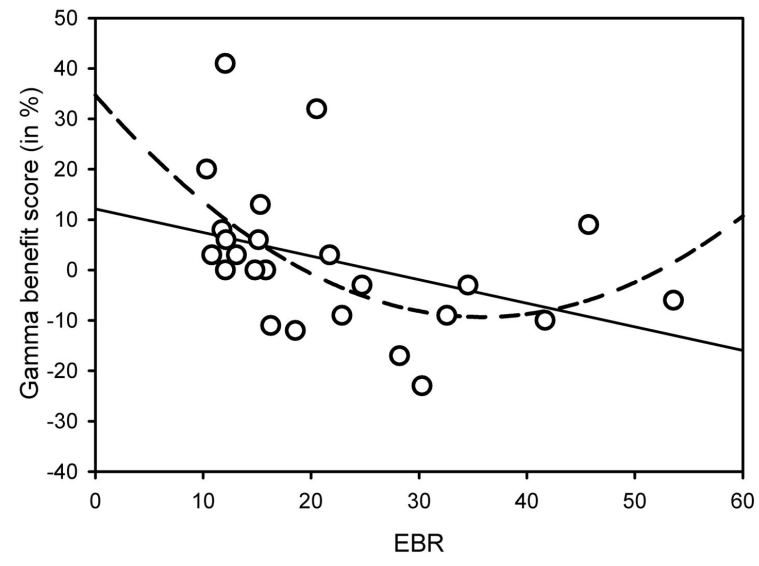

FIGURE 2 | Linear (solid line) and quadratic (dotted line) relationships between gamma-frequency $A B$ benefit scores on lag 3 and EBR [left-hand graph - linear: $F(1,22)=3.89, p=0.061$; quadratic: $F(2$, 21) $=3.84, p=0.038]$ and alpha-frequency $A B$ benefit scores on lag 3

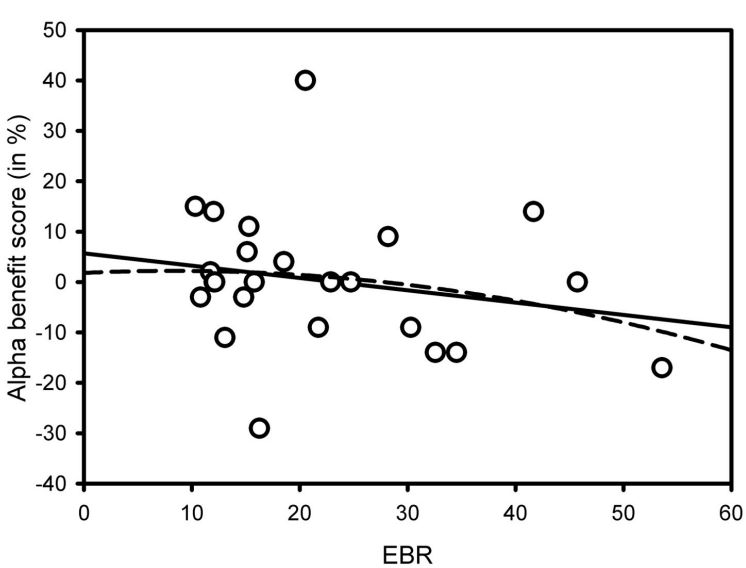

and EBR [right-hand graph - linear: $F(1,22)=1.03, p=0.32$; quadratic: $\boldsymbol{F}(\mathbf{2}, \mathbf{2 1})<\mathbf{1}$. Benefit scores were calculated by subtracting lag $3 A B$ performance in the control condition from lag $3 \mathrm{AB}$ performance in the (gamma or alpha) binaural beat condition.

resources (20). Another possible explanation is that cognitiveenhancement methods that target gamma-band activity in the brain can successfully overstimulate and thereby distract some individuals from the task, which reduces the blink (16).

Why alpha-frequency binaural beats did not affect $A B$ performance for neither the low- nor the high-EBR group remains unclear for now. It is possible, however, that all participants experienced a form of alpha entrainment in all conditions: in the $\mathrm{AB}$ task, items in the RSVP stream are typically presented with a frequency of 10 items per second, which equals visual entrainment at alpha frequency (49). Therefore, even participants in the control condition experienced some form of alpha entrainment. While it is unclear whether visual and auditory entrainment methods 
exert comparable effects on the brain (1), it cannot be ruled out that the auditory entrainment at alpha level that we presented in this study was redundant: participants already experienced alpha entrainment from the visual stimuli.

One limitation of our study is that our sample was imbalanced with respect to gender. While the available evidence does not suggest that this might have had a systematic impact on the findings, it seems to be important to replicate our results in a sample of male participants. Another limitation relates to the fact that our division of the sample in two groups based on spontaneous EBR is artificial: EBR is a continuous construct with no clear line between high and low values $(24,30)$. This division into two groups has caused its participants to share more characteristics than just EBR. For instance, sleep-deprived individuals show higher EBRs (50), so it is possible that the highEBR group featured more individuals with sleeping problems than the low-EBR group. Also, while high EBRs seem to be related to schizophrenia (30), there are individuals showing abnormally high EBRs while functioning normally (51). Unfortunately, there

\section{References}

1. Vernon D. Human Potential: Exploring Techniques Used to Enhance Human Performance. London: Routledge (2009).

2. Owen AM, Hampshire A, Grahn JA, Stenton R, Dajani S, Burns AS, et al. Putting brain training to the test. Nature (2010) 465:775-8. doi:10.1038/nature09042

3. Baas M, De Dreu C, Nijstad B. A meta-analysis of 25 years of mood-creativity research: hedonic tone, activation, or regulatory focus? Psychol Bull (2008) 134:779-806. doi:10.1037/a0012815

4. Akbari Chermahini S, Hommel B. More creative through positive mood? Not everyone! Front Hum Neurosci (2012) 6:319. doi:10.3389/fnhum.2012.00319

5. Oster G. Auditory beats in the brain. Sci Am (1973) 229:94-102. doi:10.1038/ scientificamerican1073-94

6. Turow G, Lane JD. Binaural beat stimulation: altering vigilance and mood states. In: Berger J, Turow G, editors. Music, Science, and the Rhythmic Brain: Cultural and Clinical Implications. New York, NY: Routledge (2011). p. 122-36.

7. Kuwada S, Yin TCT, Wickesberg RE. Response of the cat inferior colliculus neurons to binaural beat stimuli: possible mechanisms for sound localization. Science (1979) 206:586-8. doi:10.1126/science.493964

8. McAlpine D, Jiang D, Palmer AR. Interaural delay sensitivity and the classification of low best-frequency binaural responses in the inferior colliculus of the guinea pig. Hear Res (1996) 97:136-52. doi:10.1016/0378-5955(96)00068-8

9. Pratt H, Starr A, Michalewski HJ, Dimitrijevic A, Bleich N, Mittelman N. A comparison of auditory evoked potentials to acoustic beats and to binaural beats. Hear Res (2010) 262:34-44. doi:10.1016/j.heares.2010.01.013

10. Schwarz DWF, Taylor P. Normal and pathological oscillatory communication in the brain. Nat Rev Neurosci (2005) 6:285-96. doi:10.1038/nrn1650

11. Karino S, Yumoto M, Itoh K, Uno A, Yamakawa K, Sekimoto S, et al. Neuromagnetic responses to binaural beat in human cerebral cortex. J Neurophysiol (2006) 96:1927-38. doi:10.1152/jn.00859.2005

12. Keizer AW, Verment R, Hommel B. Enhancing cognitive control through neurofeedback: a role of gamma-band activity in managing episodic retrieval. Neuroimage (2010) 49:3404-13. doi:10.1016/j.neuroimage.2009.11.023

13. Raymond JE, Shapiro KL, Arnell KM. Temporary suppression of visual processing in an RSVP task: an attentional blink? J Exp Psychol Hum Percept Perform (1992) 18:849-60.

14. Hommel B, Kessler K, Schmitz F, Gross J, Akyürek E, Shapiro K, et al. How the brain blinks: towards a neurocognitive model of the Attentional Blink. Psychol Res (2006) 70:425-35. doi:10.1007/s00426-005-0009-3

15. Dux PE, Marois R. The attentional blink: a review of data and theory. Atten Percept Psychophys (2009) 71:1683-700. doi:10.3758/APP.71.8.1683

16. Olivers CNL, Nieuwenhuis S. The beneficial effects of additional task load, positive affect, and instruction on the attentional blink. J Exp Psychol Hum Percept Perform (2006) 32:364-79. doi:10.1037/0096-1523.32.2.364 was no way to find out whether these individuals were present in our sample and, if they were, whether they will only be found in the high-EBR group. In any case, our results suggest that the degree to which a state manipulation affects cognition depends on the individual dopamine level, a trait characteristic (30). This provides an explanation for why the current results in the field of cognitive-enhancement research are so diffuse, and hard to interpret (2). A new neurocognitive approach to cognitiveenhancement techniques should take into account characteristics that can vary between individuals, and which may play a role in how a certain enhancement technique is processed by the brain. Depending on the performance to be enhanced and the characteristics of the task, other individual differences might come into play - neural connectivity, attentional resources, and control styles are likely candidates in this respect. Identifying such candidates requires a basic understanding of the underlying functional and neural mechanisms, which suggests that successful cognitive enhancement is unlikely to work without a guiding theoretical framework.

17. Shapiro K, Schmitz F, Martens S, Hommel B, Schnitzler A. Resource sharing in the attentional blink. Neuroreport (2006) 17:163-6. doi:10.1097/01.wnr. $0000195670.37892 .1 \mathrm{a}$

18. Taatgen NA, Juvina I, Schipper M, Borst JP, Martens S. Too much control can hurt: a threaded cognition model of the attentional blink. Cogn Psychol (2009) 59:1-29. doi:10.1016/j.cogpsych.2008.12.002

19. Lagroix HEP, Spalek TM, Wyble B, Jannati A, Di Lollo V. The root cause of the attentional blink: first-target processing or disruption of input control? Atten Percept Psychophys (2012) 74:1606-22. doi:10.3758/ s13414-012-0361-5

20. Slagter HA, Davidson RJ, Lutz A. Mental training as a tool in the neuroscientific study of brain and cognitive plasticity. Front Hum Neurosci (2011) 5:17. doi:10. 3389/fnhum.2011.00017

21. Cools R. Role of dopamine in the motivational and cognitive control of behaviour. Neuroscientist (2008) 14:381-95. doi:10.1177/1073858408317009

22. Cools R, D'Esposito M. Dopaminergic modulation of flexible cognitive control in humans. In: Björklund A, Dunnet S, Iversen L, Iversen S, editors. Dopamine Handbook. Oxford, UK: Oxford University Press (2009). p. 249-60.

23. Aarts E, van Holstein M, Cools R. Striatal dopamine and the interface between motivation and cognition. Front Psychol (2011) 2:163. doi:10.3389/fpsyg.2011. 00163

24. Dreisbach G, Müller J, Goschke T, Strobel A, Schulze K, Lesch K, et al. Dopamine and cognitive control: the influence of spontaneous eyeblink rate and dopamine gene polymorphisms on perseveration and distractibility. Behav Neurosci (2005) 119:483-90. doi:10.1037/0735-7044.119.2.483

25. Colzato LS, Waszak F, Nieuwenhuis S, Posthuma D, Hommel B. The flexible mind is associated with the cathechol-O-methyltransferase (COMT) $\mathrm{Val}^{158} \mathrm{Met}$ polymorphism: evidence for a role of dopamine in the control of task-switching. Neuropsychologia (2010) 48:2764-8. doi:10.1016/j. neuropsychologia.2010.04.023

26. Colzato LS, Spapè M, Pannebakker MM, Hommel B. Working memory and the attentional blink: blink size is predicted by individual differences in operation span. Psychon Bull Rev (2007) 14:1051-7. doi:10.3758/BF03193090

27. Colzato LS, Slagter H, de Rover M, Hommel B. Dopamine and the management of attentional resources: genetic markers of striatal D2 dopamine predict individual differences in the attentional blink. J Cogn Neurosci (2011) 23:3576-85. doi:10.1162/jocn_a_00049

28. Shukla, D. Blink rate as clinical indicator. Neurology (1985) 35:286. doi:10.1212/ wnl.35.2.286

29. Blin O, Masson G, Azulay JP, Fondarai J, Serratrice G. Apomorphine-induced blinking and yawning in healthy volunteers. Br J Clin Pharmacol (1990) 30:769-73. doi:10.1111/j.1365-2125.1990.tb03848.x

30. Karson CN. Spontaneous eye-blink rates and dopaminergic systems. Brain (1983) 106:643-53. doi:10.1093/brain/106.3.643 
31. Taylor JR, Elsworth JD, Lawrence MS, Sladek JR Jr, Roth RH, Redmond DE Jr. Spontaneous blink rates correlate with dopamine levels in the caudate nucleus of MPTP-treated monkeys. Exp Neurol (1999) 158:214-20. doi:10.1006/exnr. 1999.7093

32. Freed WJ, Kleinman JE, Karson CN, Potkin SG, Murphy DL, Wyatt RJ. Eyeblink rates and platelet monoamine oxidase activity in chronic schizophrenic patients. Biological Psychiatry (1980) 15:329-32.

33. Hietala J, Syvälahti E, Vilkman H, Vuorio K, Räkköläinen V, Bergman J, et al. Depressive symptoms and presynaptic dopamine function in neuroleptic-naive schizophrenia. Schizophr Res (1999) 35(1):41-50. doi:10.1016/S0920-9964(98) 00113-3

34. Lindström LH, Gefvert O, Hagberg G, Lundberg T, Bergström M, Hartvig $\mathrm{P}$ et al. Increased dopamine synthesis rate in medial prefrontal cortex and striatum in schizophrenia indicated by L-(beta-11C) DOPA and PET. Biol Psychiatry (1999) 46(5):681-8. doi:10.1016/S0006-3223(99)00109-2

35. Colzato LS, van den Wildenberg WPM, Hommel B. Reduced spontaneous eye blink rates in recreational cocaine users: evidence for dopaminergic hypoactivity. PLoS One (2008) 2(11):e1143. doi:10.1371/journal.pone.0003461

36. Deuschel G, Goddemeier C. Spontaneous and reflex activity of facial muscles in dystonia, Parkinson's disease, and in normal subjects. J Neurol Neurosur Psychiatry (1998) 64:320-4. doi:10.1136/jnnp.64.3.320

37. Dauer W, Przedborski S. Parkinson's disease: mechanisms and models. Neuron (2003) 39:889-909. doi:10.1016/S0896-6273(03)00568-3

38. Volkow ND, Fowler JS, Wang GJ. Imaging studies on the role of dopamine in cocaine reinforcement and addiction in humans. J Psychopharmacol (1999) 13:337-45. doi:10.1177/026988119901300406

39. Colzato LS, Slagter HA, van den Wildenberg WPM, Hommel B. Closing one's eyes to reality: evidence for a dopaminergic basis of psychoticism from spontaneous eye blink rates. Pers Indiv Differ (2009) 46:377-80. doi:10.1016/j.paid. 2008.10.017

40. Gray NS, Pickering AD, Gray JA. Psychoticism and dopamine D2 binding in the basal ganglia using SPET. Pers Indiv Diff (1994) 17:431-4. doi:10.1016/ 0191-8869(94)90289-5

41. Kleven MS, Koek W. Differential effects of direct and indirect dopamine agonists on eye blink rate in cynomolgus monkeys. J Pharmacol Exp Ther (1996) 279:1211-9.

42. Groman SM, James AS, Seu E, Tran S, Clark TA, Harpster SN, et al. In the blink of an eye: relating positive-feedback sensitivity to striatal dopamine D2-Like receptors through blink rate. J Neurosci (2014) 34(43):14443-54. doi:10.1523/ JNEUROSCI.3037-14.2014
43. Colzato LS, van den Wildenberg W, Hommel B. Cognitive control and the COMT Val158Met polymorphism: genetic modulation of videogame training and transfer to task-switching efficiency. Psychol Res (2014) 78:670-8. doi:10. 1007/s00426-013-0514-8

44. Russel JA, Weiss A, Mendelsohn GA. Affect grid: a single-item scale of pleasure and arousal. J Pers Soc Psychol (1989) 57:493-502. doi:10.1037/0022-3514. 57.3.493

45. Barbato G, Ficca G, Muscettola G, Fichele M, Beatrice M, Rinaldi F. Diurnal variation in spontaneous eye-blink rate. Psychiatry Res (2000) 93:145-51. doi:10.1016/S0165-1781(00)00108-6

46. Akbari Chermahini S, Hommel B. The (b)link between creativity and dopamine: spontaneous eye blink rates predict and dissociate divergent and convergent thinking. Cognition (2010) 115:458-65. doi:10.1016/j.cognition. 2010.03.007

47. Nakatani C, Van Leeuwen C. EEG phase synchronization analysis applied to the attentional blink phenomenon. Tech Rep Atten Cogn (2004) 25:1-4.

48. Rieder MK, Rahm B, Williams JD, Kaiser J. Human gamma-band activity and behavior. Int J Psychophysiol (2011) 79:39-48. doi:10.1016/j.ijpsycho.2010.08. 010

49. Zauner A, Fellinger R, Gross J, Hanslmayr S, Shapiro K, Gruber W, et al. Alpha entrainment is responsible for the attentional blink phenomenon. Neuroimage (2012) 63:674-86. doi:10.1016/j.neuroimage.2012.06.075

50. Crevits L, Simons B, Wildenbeest J. Effect of sleep deprivation on saccades and eyelid blinking. Eur Neurol (2003) 50:176-80. doi:10.1159/000073060

51. Doughty MJ, Naase T. Further analysis of the human spontaneous eye blink rate by a cluster analysis-based approach to categorize individuals with 'normal' versus 'frequent' eye blink activity. Eye Contact Lens (2006) 32:294-9.

52. Colzato LS, Slagter HA, Spapé MA, Hommel B. Blinks of the eye predict blinks of the mind. Neuropsychologia (2008) 46:3179-83. doi:10.1016/j. neuropsychologia.2008.07.006

Conflict of Interest Statement: The authors declare that the research was conducted in the absence of any commercial or financial relationships that could be construed as a potential conflict of interest.

Copyright (c) 2015 Reedijk, Bolders, Colzato and Hommel. This is an open-access article distributed under the terms of the Creative Commons Attribution License (CC $B Y)$. The use, distribution or reproduction in other forums is permitted, provided the original author(s) or licensor are credited and that the original publication in this journal is cited, in accordance with accepted academic practice. No use, distribution or reproduction is permitted which does not comply with these terms. 